\title{
A influência da atividade física nos índices de positividade e orientação de vida no idoso
}

\section{The influence of physical activity on the positivity and life orientation indices among the elderly}

\begin{abstract}
RESUMO
O objetivo geral deste estudo foi comparar os níveis de atividade física com os níveis de Positividade e de Orientação de Vida nos idosos. A amostra foi constituída por 204 idosos com idades compreendidas entre os 61 e os 98 anos $(M=73.4 ; D P=8.42)$. Para os propósitos do presente estudo utilizamos o Escala de Positividade e Teste de Orientação de vida. Os dados foram recolhidos em diversas instituições e residências. A descrição da amostra foi realizada através das medidas de tendência central e de dispersão. Foi realizado o teste de Kolmogorov Smirnov, os testes não paramétricos de Mann-Whitney e de Kruskal-Wallis, testes de comparações múltiplas e o coeficiente de correlação de Spearman. Os resultados obtidos demonstram uma relação positiva entre atividade física e as variáveis dependentes Positividade e Orientação de Vida. Face aos resultados concluímos que a atividade física promove benefícios para o idoso idoso, nomeadamente ao nível do ganho da saúde física, bem-estar psicológico, positividade e orientação de vida.
\end{abstract}

Palavras-chave: atividade física, positividade, orientação de vida

\begin{abstract}
The purpose of this study was to compare physical activity levels with the levels of Positivity and Life Orientation in the elderly. The sample consisted of 204 older adults aged 61 and 98 years $(M=73.4$; $S D=8.42)$. For this study, we used the Positivity Scale and Life Orientation Test. Data was gathered in several institutions and residential houses homes. The description of the sample was performed through measures of central tendency and dispersion. The Kolmogorov Smirnov, nonparametric Mann-Whitney and Kruskal-Wallis, multiple comparisons, and Spearman correlation coefficient were performed. The results obtained demonstrate a positive relationship between physical activity and the dependent variables Positivity and Life Orientation. Given the results, we conclude that physical activity promotes benefits for the elderly, namely physical health gains, psychological well-being, positivity, and life guidance.
\end{abstract}

Keywords: physical activity, positivity, life orientation 
Atualmente ocorre um aumento do envelhecimento populacional a nível mundial, este facto resulta do desenvolvimento da própria sociedade (Calçada, 2009). As Nações Unidas declaram que em 2050, aproximadamente $325 \%$ da população terá 65 anos ou mais (Demirkan, 2007).

O envelhecimento caracteriza-se como um processo dinâmico do qual resultam alterações psicológicas, fisiológicas, bioquímicas e morfológicas, que progressivamente minimizam a capacidade de adaptação do idoso ao meio ambiente (Ferreira, Maciel, Silva, Santos, \& Moreira, 2010). No decorrer do envelhecimento sucede-se uma decadência não linear da capacidade funcional de vários sistemas, caracterizada particularmente pela redução e modificação da constituição muscular, da massa óssea e do sistema cardiorrespiratório, comprometendo até a execução das mais básicas tarefas diárias (Hardy \& Grogan, 2009).

Nas idosas, o sistema ósseo ressente alterações hormonais devidas ao aparecimento da menopausa, da qual podem advir doenças ósseas como a osteoporose (redução da massa óssea e deterioração da micro arquitectura) que aumenta a fragilidade óssea (Navega \& Oishi, 2007). Vidmar, et al. (2011) distinguiram três variáveis que influenciam o envelhecimento, nomeadamente: a genética, as patologias e o sedentarismo; argumentando que estas variáveis se encontram intimamente ligadas à qualidade e orientação de vida do idoso. O envelhecimento ativo diz respeito a um equilíbrio bio-psico-social, pois apesar de idoso, o indivíduo ainda é capaz de desenvolver as suas potencialidades (Ferreira, et al., 2010).

Grande parte da literatura associa um estilo de vida ativo à positividade e à prevenção e diminuição dos danos subjacentes ao processo de envelhecimento (Rocha \& Freire, 2007). A atividade física consiste em qualquer movimento corporal que demande gasto energético acima dos níveis de repouso, pode ser vivenciada nas dimensões do lazer, transporte, desporto, atividades domésticas e ocupacionais.

A influência de aspetos psicológicos, como a positividade, a autoeficácia, a distração e a interação social e aspetos fisiológicos, através do acréscimo da transmissão sináptica de endorfinas e da condutância pós-sináptica de potássio, e da neurogénese (Kempermann et al., 2010), são ainda aspetos a terem em consideração no estudo da atividade física em idosos. Os benefícios biológicos resultantes da prática de atividade física manifestam um efeito dose-resposta, uma vez que a sobrecarga física à qual o organismo é sujeito causa alterações em órgãos e sistemas variando conforme a intensidade, a frequência e a duração do estímulo. Os idosos sedentários, que iniciem a prática de atividade física, mesmo que diminuta, podem logo obter alguns dos benefícios a esta associados (Assumpção, et al., 2008).

A prática regular de atividade física faculta alterações de funções cerebrais que promovem a prevenção e o tratamento da depressão, obesidade, cancro, doenças neurológicas, acidentes vasculares cerebrais, lesões na medula espinhal e minimização de danos cognitivos inerentes ao envelhecimento (Dishman et al., 2006).

A atividade física é um meio eficiente e económico (Santos \& Knijnik, 2006), responsável pela qualidade e orientação de vida dos idosos, associando-se ao prazer de viver e à positividade (Carvalho \& Madruga, 2011). Tendo em conta os benefícios que a atividade física provoca na saúde do indivíduo, esta pode ser considerada um método eficaz na melhoria da saúde pública (Scherer, et al, 2007). Deste modo, a utilização de serviços de saúde e de medicamentos, podem ser minimizados através da melhoria da qualidade de saúde e de vida proporcionada pela prática de atividade física (Zaitune et al., 2010).

Estudos demonstram que idosos fisica- 
mente ativos ostentam uma menor prevalência de doenças mentais e um maior nível de positividade, do que os idosos fisicamente não ativos (Benedetti et al., 2008). Os benefícios da prática de atividade física e a sua influência ao nível da positividade são há muito conhecidos. Contudo, apenas uma pequena percentagem de idosos pratica atividade física com regularidade (Benedetti et al., 2008).

Existe uma crescente preocupação em adquirir ou manter hábitos saudáveis, que promovam a longevidade e a positividade dos idosos. Uma vez que o tédio, a inatividade e a expectativa de enfermidade são os principais fatores responsáveis pela decadência da autonomia funcional do idoso (Santos \& Knijnik, 2006). Perez, et al. (2010) visam que a autonomia possibilita ao idoso administrar a sua vida consoante as suas necessidades. De acordo com este fato, Duca, et al. (2012) afirmam que para o bem-estar do idoso, o convívio familiar e comunitário são essenciais, a fim de lhes proporcionar cidadania, dignidade, positividade e autonomia.

Paskulin, et al. (2010) concluíram que a qualidade de vida tem significados específicos para a população idosa, como: ter saúde, viver bem, boa convivência quer com a família, quer com os amigos, ter o que comer, ter uma alimentação saudável, poder realizar atividades de lazer e ter recursos para sustentar as suas necessidades. A satisfação com a vida promove a positividade e influencia a orientação de vida, sendo entendida como uma avaliação genérica das circunstâncias da própria vida do indivíduo de acordo com um critério por ele designado. Pode considerar-se um juízo cognitivo de áreas específicas da vida, como: a saúde, a autonomia, as relações sociais, o trabalho, entre muitos outros (Joia, et al., 2007). Devido ao progressivo aumento da população idosa, torna-se necessário proporcionar-lhes (para além de uma maior longevidade) positividade, qualidade de vida e satisfação pessoal.
O presente estudo teve como objetivo geral comparar os níveis de atividade física com a Positividade e a Orientação de Vida dos idosos, ou seja, de que forma a prática de atividade física influencia os diferentes níveis de Positividade e Orientação de Vida na população idosa. Já os objetivos específicos consistiram em:

I. Comparar por idade os índices da atividade física, da Orientação de vida e da Positividade no idoso;

II. Comparar os sexos relativamente à influência da prática de atividade física com a Positividade e a Orientação de Vida;

III. Comparar por local de residência os índices da atividade física, da Orientação de vida e da Positividade no idoso;

IV. Comparar por situação profissional os índices da atividade física, da Orientação de vida e da Positividade no idoso;

V. Comparar por estado civil os índices da atividade física, da Orientação de vida e da Positividade no idoso;

VI. Comparar por habilitações literárias os índices da atividade física, da Orientação de vida e da Positividade no idoso;

VII. Comparar por IMC os índices da atividade física, da Orientação de vida e da Positividade no idoso.

Assim, a hipótese espectável diz respeito à existência de uma influência significativa da atividade física sobre a positividade e a orientação de vida dos idosos.

\section{MÉTODOS}

O presente estudo caracteriza-se como uma investigação quantitativa, evocando como desígnio geral a descrição e observação entre variáveis através da examinação das mudanças ocorridas na variável dependente após a manipulação das variáveis independentes; transversal, pois os dados serão recolhidos numa única circunstância (Freixo, 2011). Esta investigação é de cariz quasi-experimental, pois os pesquisadores não 
exercem qualquer tipo de controlo sobre os níveis da variável independente e recorrendo a estas variáveis pretende-se observar o efeito sobre as variáveis dependentes.

As variáveis independentes a analisar são: a prática do exercício físico, o sexo, a idade, o estado civil, o local de residência, as habilitações literárias e a situação profissional. As variáveis dependentes são a Positividade e a Orientação de vida.

\section{Amostra}

A amostra foi constituída por 204 idosos, 128 (62.7\%) do sexo feminino e 76 (37.3\%) do sexo masculino, com idades compreendidas entre os 61 e os 98 anos $(M=73.4 ; D P=8.42) .135$ $(66.2 \%)$ idosos viviam em residência própria, os restantes 69 (33.8\%) viviam em instituições asilares. Quanto ao estado civil 18 idosos (8.8\%) são solteiros, 97 (47.5\%) são casados e os restantes (43.6\%) viúvos. A maior parte dos idosos questionados têm o ensino básico (76.5\%), 38 (18.6\%) terminaram o ensino secundário e apenas 10 idosos (4.9\%) têm ensino superior. Relativamente à classificação dos indivíduos quanto ao seu nível de atividade física, esta seguiu os seguintes critérios: os indivíduos que praticam atividade física zero dias por semana foram considerados inativos, os que praticam atividade física somente um dia por semana foram designados como pouco ativos, os idosos que praticam atividade física dois a três dias por semana foram classificados como ativos, e por fim, denominaram-se por muito ativos os indivíduos que afirmaram praticar atividade física mais de três dias por semana.

Relativamente à situação profissional registaram-se 186 (91.2\%) idosos reformados e $18(8.8 \%)$ empregados. No que diz respeito à prática de exercício físico numa semana normal, 57 (27.9\%) idosos não praticam atividade física, 44 (21.6\%) praticam apenas uma vez por semana, 61 (29.9\%) praticam duas a três vezes por semana e $42(20.6 \%)$ praticam atividade física mais de três vezes por semana.
Os indivíduos admitidos na investigação tiveram como critério de inclusão: ter idade superior a 60 anos, e como critérios de exclusão: ter idade inferior a 60 anos, serem portadores de patologia que lhes impossibilitasse a leitura e preenchimento dos questionários assim como o preenchimento incorreto dos questionários e a desistência do participante por sua própria vontade.

\section{Instrumentos}

Os dados foram recolhidos através da aplicação de quatro questionários de autopreenchimento: o Questionário Sócio-biográfico, o Questionário da Atividade Física, a Escala da Positividade e o Teste da Orientação de Vida (LOT - Life Orientation Test).

O Questionário Sócio-biográfico foi realizado pela equipa de pesquisa, sendo constituído pelos seguintes itens: idade, sexo, peso, altura, estado civil, local de residência, habilitações literárias e situação profissional e que constituíram como variáveis independentes. O Questionário da Atividade Física, composto por apenas dois itens foi aplicado à amostra, a fim de verificar a frequência com que os indivíduos praticam atividade física.

A Escala da Positividade é constituída por 8 itens (frases) que avaliam a Positividade (visão positiva) dos indivíduos para com a sua vida (Caprara et al., 2012). Esta escala contém quatro respostas possíveis para cada item, nomeadamente: 1- Discordo Totalmente, 2- Discordo, 3 -Concordo e 4- Concordo Totalmente. Esta Escala foi validada na Itália, no Japão, U.S.A e Espanha (Yamaguchi, et al., 2008). A Escala utilizada foi traduzida para uma versão portuguesa para possibilitar a sua aplicação à amostra.

O Teste da Orientação de Vida foi validado na Tailândia (Li, 2012). Para ser utilizado no presente estudo foi traduzido para uma versão portuguesa. Está organizado em 8 itens (4 itens positivos -1 , 3, 4, 7 e 4 itens negativos -2 , 5, $6,8)$ que avaliam a orientação de vida dos indivíduos. Esta escala contém igualmente quatro 
respostas possíveis para cada item, nomeadamente: 1- Discordo Totalmente, 2- Discordo, 3 -Concordo e 4- Concordo Totalmente.

Para atribuir um valor que meça a Escala da Positividade e o Teste de Orientação de Vida, foram utilizadas as somas das pontuações obtidas a partir das oito variáveis subjacentes a cada instrumento, podendo a pontuação de cada indivíduo variar entre os 8 e os 32 pontos.

\section{Procedimentos}

Foi estabelecido contacto com três lares de idosos, e após a obtenção do consentimento de cada instituição, foram entregues os questionários.

A outra parte da amostra, que habitava em residência própria, foi selecionada aleatoriamente, os indivíduos foram abordados na rua e nas suas residências e sensibilizados para a participação neste estudo, através do preenchimento dos questionários.

Cada questionário continha um pedido de autorização individual para a realização deste estudo, no qual estava especificado o seu objetivo, e ainda garantido total anonimato e confidencialidade. Em cada questionário, juntamente com as instruções de preenchimento também foi evidenciada a importância da total sinceridade e naturalidade na resposta às questões.

\section{Análise Estatística}

Para o tratamento dos resultados foi utilizado o programa estatístico SPSS - Statistical Package for Social Sciences, na sua versão 19.0 para o sistema Windows.

A descrição da amostra foi realizada através de medidas de tendência central: média, mediana, moda; e de dispersão: desvio-padrão. Foi realizado o teste de Kolmogorov -Smirnov para verificar a normalidade dos dados, tendo-se concluído que as variáveis dependentes não apresentavam uma distribuição normal em quase todos os grupos considerados. Como não se verificou o pressuposto da normalidade dos dados para a realização de testes paramétricos, foram utilizados os testes não paramétricos de Mann-Whitney, para comparar dois grupos, e de Kruskal-Wallis quando se pretendeu compara três ou mais grupos.

Nos casos em que após a utilização do teste de Kruskal-Wallis (1-way ANOVA) se obtiveram resultados com diferenças significativas, foram realizados testes de comparações múltiplas (Marôco, 2010). Utilizou-se o coeficiente de correlação de Spearman, a fim de estudar a correlação entre as variáveis.

\section{RESULTADOS}

Para estudar a correlação entre as variáveis idade, Índice de Positividade (IP), Índice de Orientação de Vida (IOV), índice de Atividade Física numa Semana Normal (IAFN), índice de Atividade Física na Última Semana, foi utilizado o coeficiente de correlação de Spearman.

A associação encontrada entre a idade e o IAFN $\left(r^{2}=-.316\right)$ e a idade e o índice de Atividade Física na Última Semana $\left(r^{2}=-.240\right)$ não foi estatisticamente significativa. O IP está fortemente correlacionado com o IOV $\left(r^{2}=.630\right)$. Verificou-se uma correlação positiva fraca do IP com o IAFN $\left(r^{2}=.178\right)$ e com o índice de Atividade Física na Última Semana $\left(r^{2}=.191\right)$. De igual modo, o IOV está positivamente correlacionado com o IAFN $\left(r^{2}=.164\right)$ e com o índice de Atividade Física na Última Semana $\left(r^{2}=\right.$ .206). Verificou-se, assim, que quanto maior a frequência da prática de atividade física do idoso, mais elevados são os seus níveis de positividade e orientação de vida.

Para avaliar a influência da atividade física na Positividade e na Orientação de Vida, objetivo principal do estudo, uma vez que não se verificou a normalidade dos dados, foram utilizados os testes de Kruskal-Wallis, com os quais se verificaram diferenças significativas relativamente ao IAFN tanto no IP $\left(\chi_{(3)}^{2}=40.929 ; p=.001\right)$ como no IOV $\left(\chi_{(3)}^{2}=37.000 ; p=.001\right)$. Os valores das médias evidenciaram que os valores 
mais baixos na variável IP foram obtidos nos indivíduos que não praticavam exercício físico (IP, $M=19.19$; IOV, $M=20.56$ ) e os mais altos nos que praticavam entre duas a três vezes por semana (IP, $M=23.79$; IOV, $M=24.30$.

Após a utilização dos testes de comparações múltiplas, verificaram-se diferenças significativas, quanto à variável IP, entre os grupos que praticavam atividade física: 0 vezes/semana - 1 vez/semana $(t=-34.534 ; p=.021)$, 0 vezes/ semana - 2 a 3 vezes/semana $(t=-64.188 ; p$ $=.001)$ e 0 vezes/semana -+3 vezes $/$ semana $(t$ $=-58.150 ; p=.001)$; e quanto à variável Orientação de Vida, entre os grupos: 0 vezes/semana -+3 vezes/semana $(t=-54.622 ; p=.001), 0$ vezes/semana -2 a 3 vezes/semana $(t=-57.713$; $p=.001), 1 \mathrm{vez} / \mathrm{semana}-+3$ vezes $/$ semana $(t=$ - 36.131; $p=.026)$ e 1 vez/semana -2 a 3 vezes/ semana $(t=-39.221 ; p=.004)$.

Relativamente ao índice de Atividade Física na Última Semana, também se verificaram diferenças significativas na Positividade $\left(\chi^{2}{ }_{(3)}=\right.$ $44.129 ; p=.001)$ e na Orientação de Vida $\left(\chi^{2}{ }_{(3)}\right.$ $=43.846 ; p=.001)$. As médias mais baixas foram obtidas no grupo dos que não praticavam e no caso do IP foi entre os que praticam duas a três vezes por semana, assim na variável IOV. As médias mais altas foram, no caso do IP nos que praticam 2 a 3 vezes por semana e nos que praticam mais de 3 vezes no caso do IOV.

$\mathrm{Na}$ análise comparativa que foi realizada quanto à Positividade, verificaram-se diferenças significativas entre os grupos: 0 vezes/semana-1 Quadro 1

Diferenças quanto ao local de residência. vez/semana $(t=-47.201 ; p=.001)$, 0 vezes/ semana- $2 / 3$ vezes/semana $(t=-61.519 ; p=$ $.001)$ e 0 vezes/semana- +3 vezes/semana $(t$ $=-53.169 ; p=.001)$. Quanto à variável IOV, os testes de comparações múltiplas mostraram diferenças significativas entre os grupos: 0 vezes/semana-1 vez/semana $(t=-37.954 ; p=$ $.005), 0$ vezes/semana- $2 / 3$ vezes/semana $(t=$ $58.747 ; p=.001)$ e 0 vezes/semana +3 vezes/ semana $(t=-65.084 ; p=.001)$. Verificando-se que quanto maior a frequência da prática de atividade física, mais elevados são os níveis de positividade e orientação de vida.

Foi utilizado o teste Mann-Whitney para verificar se existem diferenças significativas nas variáveis: IP, IOV, IAFN e índice de Atividade Física na Última Semana quanto ao sexo, local de residência e situação profissional.

Quanto às comparações por sexo verificaram-se diferenças significativas apenas na variável índice de Atividade Física na Última Semana (IAFU) $(\mathrm{Z}=-2.154 ; p=.031)$, em que os homens apresentam um maior índice comparativamente às mulheres.

Após a utilização do teste de Mann-Whitney, verificou-se que o local de residência (Quadro 1) influencia significativamente a Positividade $(Z=-4.392 ; p=.001)$, a Orientação de Vida $(Z=-4.177 ; p=.001)$ e os índices de Atividade Física numa Semana Normal $(Z=-3.150$; $p=.002)$ e na Última Semana $(Z=-3.445 ; p$ $=.001)$. Obtendo-se valores superiores para os indivíduos que vivem em residência própria.

\begin{tabular}{|c|c|c|c|c|c|}
\hline & $\begin{array}{c}\text { Residência } \\
\text { própria } \\
(n=135) \\
M\end{array}$ & $\begin{array}{c}\text { Instituição } \\
(n=69) \\
M\end{array}$ & $U$ & $Z$ & $p$ \\
\hline IP & 22.96 & 20.22 & 2911.500 & -4.392 & .001 \\
\hline IOV & 23.47 & 21.16 & 2988.500 & -4.177 & .001 \\
\hline IAFN & 2.22 & 1.35 & 3426.500 & -3.150 & .002 \\
\hline IAFU & 1.79 & .90 & 3334.500 & -3.445 & .001 \\
\hline
\end{tabular}

$U$ e $Z$ - estatísticas do teste de Mann Whitney; $M$ - Média; $p$ - valor de significância; IP - Índice de Positividade; IOV - Índice de Orientação de Vida; IAFN - Índice de Atividade Física numa Semana Normal; IAFU - Índice de Atividade Física na Última Semana. 
Verificou-se que a situação profissional (Quadro 2) influencia significativamente o IAFN $(Z=-3.408 ; p=.001)$ e o índice de
Atividade Física na Última Semana $(\mathrm{Z}=$ $2.821 ; p=.005)$. Verificando-se valores superiores para os indivíduos reformados.

Quadro 2

Diferenças quanto à situação profissional.

\begin{tabular}{lccccc}
\hline & $\begin{array}{c}\text { Empregado } \\
(n=18)\end{array}$ & $\begin{array}{c}\text { Reformado } \\
(n=186) \\
M\end{array}$ & $\boldsymbol{M}$ & $\boldsymbol{U}$ & $\boldsymbol{Z}$ \\
\hline IP & 22.78 & 21.96 & 1472.500 & -.845 & .398 \\
IOV & 22.83 & 22.68 & 1658.001 & -.067 & .946 \\
IAFN & 3.22 & 1.80 & 875.500 & -3.408 & .001 \\
IAFU & 2.33 & 1.40 & 1024.500 & -2.821 & .005 \\
\hline
\end{tabular}

$U$ e $Z$ - estatísticas do teste de Mann Whitney; $M$ - Média; $p$ - valor de significância; IP - Índice de Positividade; IOV - Índice de Orientação de Vid̦a; IAFN - Índice de Atividade Física numa Semana Normal; IAFU - Índice de Atividade Física na Última Semana.

Para estudar as diferenças quanto ao estado civil, habilitações literárias, situação profissional e IMC, foi realizado o Teste Kruskal-Wallis.

Quanto ao estado civil verificou-se que este influencia significativamente todas as variáveis. $\mathrm{Na}$ análise comparativa realizada quanto ao estado civil verificaram-se diferenças significativas entre os casados e os viúvos relativamente aos índices de Positividade $(t=37.188$; $p=.001)$, Orientação de Vida $(t=22.284 ; p=$ .029), Atividade Física numa Semana Normal $(t=29.190 ; p=.002)$ e Atividade Física na Última Semana $(t=29.713 ; p=.001)$.

Quadro 3

Diferenças quanto ao estado civil.

\begin{tabular}{lccccc}
\hline & $\begin{array}{c}\text { Solteiro } \\
(n=18)\end{array}$ & $\begin{array}{c}\text { Casado } \\
(n=97)\end{array}$ & $\begin{array}{c}\text { Viúvo } \\
(\boldsymbol{n}=89)\end{array}$ & $\boldsymbol{\chi}_{(2)}$ & $p$ \\
\hline IP & $\boldsymbol{M}$ & $\boldsymbol{M}$ & $\boldsymbol{M}$ & & \\
IOV & 22.22 & 23.29 & 20.63 & 18.573 & .001 \\
IAFN & 22.44 & 23.44 & 21.92 & 7.068 & .029 \\
IAFU & 2.67 & 2.28 & 1.39 & 14.566 & .001 \\
\hline
\end{tabular}

$U$ e $Z$ - estatísticas do teste de Mann Whitney; $M$ - Média; $p$ - valor de significância; IP - Índice de Positividade; IOV - Índice de Orientação de Vida; IAFN - Índice de Atividade Física numa Semana Normal; IAFU - Índice de Atividade Física na Última Semana.

Quanto às habilitações literárias verificaram-se diferenças significativas no IP $\left(\chi_{(2)}^{2}=6.593\right.$; $p=.037)$, IAFN $\left(\chi_{(2)}^{2}=16.423 ; p=.001\right) \mathrm{e}$ no índice de Atividade Física na Última Semana $\left(\chi_{(2)}^{2}=11,462 ; p=.001\right)$. Os testes de comparações múltiplas realizados permitem verificar diferenças significativas entre idosos com ensino básico e ensino superior $(t=-27,277$; $p=.031)$ na variável IP, IAFN $(t=-50.099 ; p$ $=.024)$ e índice de Atividade Física na Última Semana $(t=-27.913 ; p=.020)$. As médias mais baixas foram obtidas nos indivíduos com o menor nível de escolaridade e as mais altas foram: IP - Ensino secundário (23.63), No ensino Superior foi no IOV (23.90), IAFN (3.10) e IAFU (2.60). 
Quadro 4

Diferenças quanto às habilitações literárias.

\begin{tabular}{|c|c|c|c|c|c|}
\hline & $\begin{array}{c}\text { Ens. Básico } \\
\begin{array}{c}(n=156) \\
M\end{array}\end{array}$ & $\begin{array}{l}\text { Ens. Secundário } \\
\qquad \begin{array}{c}(n=38) \\
M\end{array}\end{array}$ & $\begin{array}{c}\text { Ens. Superior } \\
\left(\begin{array}{c}(n=10) \\
M\end{array}\right.\end{array}$ & $\chi_{(2)}^{2}$ & $p$ \\
\hline IP & 21.63 & 23.63 & 22.30 & 6.593 & .037 \\
\hline IOV & 22.40 & 23.58 & 23.90 & 2.911 & .233 \\
\hline IAFN & 1.63 & 2.82 & 3.10 & 16.423 & .001 \\
\hline IAFU & 1.22 & 2.26 & 2.60 & 11.462 & .001 \\
\hline
\end{tabular}

$\chi^{2}$ - estatística do teste de Kruskal-Wallis; $M$ - Média; $p$ - valor de significância; IP - Índice de Positividade; IOV - Índice de Orientação de Vid̦a; IAFN - Índice de Atividade Física numa Semana Normal; IAFU - Índice de Atividade Física na Última Semana.

Relativamente à classificação do índice de massa corporal (IMC), esta seguiu os seguintes critérios: os indivíduos cujo IMC era menor que 18.5 foram considerados abaixo do peso, os que tinham o seu IMC entre 18.6 e 24.9 foram designados como tendo o peso normal, os idosos com IMC entre 25 e 29.9 foram classificados como tendo excesso de peso, e os indivíduos com um IMC superior a 30 foram classificados como obesos.

Quanto ao índice de massa corporal (IMC) não se verificaram diferenças significativas.

Quadro 5

Diferenças quanto ao IMC.

\begin{tabular}{|c|c|c|c|c|c|c|}
\hline & $\begin{array}{l}\text { Abaixo do peso } \\
\qquad \begin{array}{c}(n=1) \\
M\end{array}\end{array}$ & $\begin{array}{c}\text { Normal } \\
(n=83) \\
M\end{array}$ & $\begin{array}{c}\text { Excesso de peso } \\
\qquad(n=108) \\
M\end{array}$ & $\begin{array}{c}\text { Obesidade } \\
(n=12) \\
M\end{array}$ & $\chi_{(2)}^{2}$ & $p$ \\
\hline IP & 22.00 & 22.04 & 22.31 & 19.50 & 2.681 & .443 \\
\hline IOV & 23.00 & 22.59 & 22.83 & 22.08 & 0.989 & .804 \\
\hline IAFN & 3.00 & 2.14 & 1.81 & 1.33 & 4.714 & .194 \\
\hline IAFU & 3.00 & 1.59 & 1.41 & 1.33 & 2.637 & .451 \\
\hline
\end{tabular}

$\chi^{2}$ - estatística do teste de Kruskal-Wallis; $M$ - Média; $p$ - valor de significância; IP - Índice de Positividade; IOV - Índice de Orientação de Vid̦a; IAFN - Índice de Atividade Física numa Semana Normal; IAFU - Índice de Atividade Física na Última Semana.

\section{DISCUSSÃO}

Através dos resultados obtidos foi possível concluir que a prática de atividade física influencia positivamente os índices de Orientação de vida e de Positividade dos idosos, corroborando a hipótese expectável. E ainda, que estes dois índices se influenciam fortemente entre si. Tal como é visível no estudo de Joia, et al. (2007) a associação entre qualidade e satisfação com a vida com a dependência e a autonomia do idoso podem ser muito beneficiadas pela prática de atividade física. De acordo com este facto também se encontra o estudo de Benedetti, et al. (2008) realizado com 875 idosos, que concluiu que os níveis de atividade física influenciam o estado de saúde mental e a positividade, evidenciando uma menor presença de sintomas negativos nos indivíduos não sedentários.

Não foi verificada influência da idade na frequência da prática de atividade física da população em estudo. Este facto está de acordo com Joia et al. (2007) que afirmam que a idade não é um fator determinante de condição física ou psicológica, pois parte da população idosa ostenta prematuramente declínio no 
seu estado de saúde e nas suas competências cognitivas, enquanto outros vivem sadios até idades muito avançadas.

Em discordância com o estudo de Zaitune et al. (2010) realizado com 250 idosos cujos resultados obtidos foram uma maior prevalência de idosos do sexo feminino ativos globalmente, neste estudo não se verificou grande diferença entre os sexos relativamente à prática geral de atividade física.

Foi observada uma grande influência do local onde os idosos residem nos seus índices de Positividade, Orientação de Vida e prática de atividade física, constatando-se que indivíduos que vivem em residência própria têm níveis de Positividade, Orientação de Vida e atividade física superiores aos que se encontram institucionalizados. Esta conclusão vai de encontro ao resultado de um estudo elaborado por Duca, et al. (2012) onde se verificou que a institucionalização se encontra intimamente ligada à falta de prática de atividade física, à positividade e à orientação de vida o idoso.

Quanto à situação profissional concluiu-se que indivíduos reformados apresentam uma maior prática de atividade física que os restantes, isto pode dever-se ao facto da amostra ser constituída maioritariamente por idosos reformados. Notou-se uma forte influência do estado civil do idoso nos seus níveis de Positividade, Orientação de Vida e atividade física, principalmente nos casados e viúvos. A literatura sustenta a ideia de que as mulheres vivem mais tempo que os homens, esta ocorrência pode dever-se à propensão das idosas a sofrerem problemas de saúde crónicos e incapacitantes, enquanto os idosos tendem a desenvolver doenças fatais de curta duração (Papalia, et al., 2006). Outro fundamento associado à maior longevidade das mulheres, são os fatores socioculturais, que evidenciam de um modo geral, que as mulheres se casam com homens com idade superior à sua, gerando assim um número superior de viúvas; além de que as mulheres separadas e viúvas têm uma tendência maior ao isolamento, e consequentemente a menores níveis de positividade, pois voltam a casar com menor frequência relativamente aos homens que se encontram nas mesmas condições.

As habilitações literárias influenciam diretamente o nível de Positividade e de prática de atividade física dos idosos com ensino básico ou superior, curiosamente não se verificou influência desta sobre a orientação de vida do individuo.

Com o crescente aumento populacional dos idosos, torna-se essencial a formação de estratégias que garantam uma boa qualidade de vida na velhice a fim de promover elevados índices de Positividade e Orientação de vida.

\section{CONCLUSÃO}

O presente estudo comprovou que a atividade física influencia os índices de Positividade e Orientação de Vida da população idosa corroborando a literatura especializada nesta temática. Foi observada uma grande influência do local onde os idosos residem nos índices de Positividade, Orientação de Vida e prática de atividade física, constatando-se que indivíduos que vivem em residência própria têm níveis de Positividade e Orientação de vida superiores aos que se encontram institucionalizados, assim como um índice de prática de atividade física mais elevado.

Relativamente à situação profissional, indivíduos reformados apresentam uma maior prática de atividade física que os restantes. Os idosos casados e viúvos têm níveis de Positividade, Orientação de Vida e atividade física mais elevados, quando comparados com os solteiros, evidenciando a forte influência do estado civil no idoso.

No que diz respeito às habilitações literárias, verificou-se que têm grande influência sobre o nível de Positividade e de prática de atividade física dos idosos com ensino básico ou superior. A atividade física providencia muitos benefícios à saúde do idoso, e consequentemente ao seu 
nível de positividade, satisfação pessoal e orientação de vida. Tornando a atividade física uma ótima estratégia na melhoria da saúde física e psicológica do indivíduo.

Agradecimentos:

Nada declarado.

\section{Conflito de Interesses:}

Nada declarado.

\section{Financiamento:}

Nada declarado.

\section{REFERÊNCIAS}

Assumpção, C., Pellegrinotti, I., Neto J., \& Montebelo, M. (2008). Controle da intensidade regressiva de exercícios localizados em mulheres idosas por meio a percepção subjetiva de esforço (BORG). Revista de Educação Física, 19, 33- 39

Benedetti, T., Borges, L., Petroski, E. \& Gonçalves, L. (2008). Atividade física e estado de saúde mental de idosos. Revista de Saúde Pública, 42(2), 302-307. https://doi.org/10.1590/ S0034-89102008005000007

Calçada, J. (2009). Turismo Sênior: (Re) Contextualização das clientelas turísticas seniores. Revista Transdisciplinar de Gerontologia, 3(1), 46-63. https://doi. org/10.5016/1980-6574.2011v17n2p328

Caprara, G., Alessandri, G., Eisenberg, N., Kupfer, A., Steca, P., Caprara, M., Yamaguchi, S., Fukuzawa, A., \& Abela, J. (2012). The positivity scale. Psychological Assessment. Advance online publication. https://doi. org/10.1037/a0026681

Carvalho, R. \& Madruga, V. (2011). Envelhecimento e prática de atividade física: a influência do género. Motriz, 17 (2), 328-337. https://doi.org/10.5016/19806574.2011v17n2p328

Demirkan, H. (2007). Housing for the aging population. European Review of Aging and
Physical Activity (EURAPA), 4(1), 33-38. https://doi.org/10.1007/s11556-007-0016-z Dishman, R., Berthoud, H., Booth F., Cotman, C., Edgerton V., \& Fleshner, M. (2006). Neurobiology of exercise. Obesity, 14(3), 345-56. https://doi.org/ 10.1038/oby.2006.46

Duca, G., Silva, S., Thumé, E., Santos, I., \& Hallal, P. (2012). Indicadores da institucionalização de idosos: estudo de casos e controles. Revista de Saúde Pública, 46(1), 147-153. https://doi. org/ 10.1590/S0034-89102012000100018

Ferreira, O., Maciel, S., Silva, A., Santos, W., \& Moreira, M. (2010). O envelhecimento ativo sob o olhar de idosos funcionalmente independentes. Revista Escola de Enfermagem USP, 44(4), 1065-1069. https://doi. org/10.1590/S0080-62342010000400030

Freixo, M. (2011). Metodologia Científica. Lisboa: Instituto Piaget.

Hardy, S. \& Grogan, S. (2009). Preventing disability through exercise: investigating older adults' influences and motivations to engage in physical activity. Journal of Health Psychology, 14, 1036-1046. https://doi. org/1010.1177/1359105309342298

Joia, L., Ruiz, T., \& Donalisio, M. (2007). Condições associadas ao grau de satisfação com a vida entre a população de idosos. Revista de Saúde Pública, 41(1), 131-138. https://doi.org/10.1590/S003489102007000100018

Kempermann, G., Fabel, K., Ehninger, D., Babu, H., Leal-Galicia, P., Garthe, A., \& Wolf, S. (2010). Why and how physical activity promotes experience-induced brain plasticity. Frontiers in Neuroscience, 4, 189. https://doi.org/10.3389/fnins.2010.00189

Li, C. H. (2012). Validation of the chinese version of the life orientation test with a robust weighted least squares approach. Psychological Assessment. Advance online publication. https://doi.org/10.1037/ a0026612

Marôco, J. (2010). Análise Estatística com o PASW Statistics. Pêro Pinheiro, Portugal: 
Report Number.

Navega, M. \& Oishi, J. (2007) Comparação da qualidade de vida relacionada à saúde entre mulheres na pós-menopausa praticantes de atividade física com e sem osteoporose. Revista Brasileira Reumatol, 47(4), 258-264. https://doi.org/10.1590/S048250042007000400004

Papalia, D., Olds, S., \& Feldman, R. (2006). Desenvolvimento Humano. Porto Alegre: Artmed.

Paskulin, L., Córdova, F., Costa, F., \& Vianna, L. (2010). Percepção de pessoas idosas sobre qualidade de vida. Acta Paul Enferm, 23(1), 101-107. https://doi.org/10.1590/S010321002010000100016

Perez, A., Tavares, O., Daltio, G., \& Farinatti,P. (2010). Estudo comparativo da autonomia de ação de idosas praticantes e não praticantes de exercícios físicos regulares. Revista Brasileira de Medicina do Esporte, 16(4), 254-258. https://doi.org/10.1590/S151786922010000400004

Rocha, S. \& Freire, M., (2007). Nível de atividade física habitual e autopercepção do estado de saúde em idosas no município de Jequié Bahia. Revista Brasileira em Promoção da Saúde. 20(3), 161-167.

Santos, S. \& Knijnik, J. (2006). Motivos de adesão à prática de atividade física na vida adulta intermediária I. Revista Mackenzie de Educação Física e Esporte, 5(1), 23-34.
Scherer, M., Andrade, S., Mello, A., \& Bonetti, A. (2007). O viver saudável e o viver não saudável: o significado para mulheres maduras e ativas. Cadernos de Saúde Coletiva, 15(1), 131-146.

Yamaguchi, S., Lin, C., Morio, H., \& Okumura, T. (2008). Motivated expression of self-esteem across cultures. In R. M. Sorrentino, R. \& S. Yamaguchi (Eds), Handbook of motivation and cognition across cultures (pp. 369-392). San Diego, C.A: Elsivier.

Vidmar, M., Potulski, A., Sachetti, A., Silveira, M., \& Wibelinger, L. (2011). A atividade Física e Qualidade de vida em idosos. Revista Saúde e Pesquisa, 4(3), 417-424.

Zaitune, M., Barros, M., César, C., Carandina, L., Goldbaum, M., \& Alves, M. (2010). Fatores associados à prática de atividade física global e de lazer em idosos: Inquérito de Saúde no Estado de São Paulo (ISASP), Brasil. Caderno de Saúde Pública, 26(8), 1606-1618. https://doi.org/10.1590/S0102311X2010000800014 